\title{
Novel In Vivo Parasite Biology-Implications for Pathogenesis
}

$\mathrm{P}$ lasmodium falciparum is responsible for millions of infections annually and can result in a range of outcomes. Infections in adults with prior exposure typically are asymptomatic and serve as reservoirs for continued infection. When children are infected, however, they can present with nonspecific flu like symptoms with a small percentage of infections resulting in severe disease and death. These clinical observations raise two questions. Why do only a small percentage of children succumb to severe disease and does this stark variation of conditions from an asymptomatic to severely ill patient impact parasite biology?

One potential explanation for diverse outcomes is the occurrence of variation in individual parasite biology. Biologic function can be inferred from the comparative analysis of transcript levels in pathways and gene ontology functions. Prior studies of whole genome transcription from in vitro cultivated isolates collected from across the globe found minimal global transcriptional differences (1). Evaluation of whole genome transcription of parasites directly from blood samples, however, demonstrated novel biology not previously seen from in vitro studies (2). Three transcriptional programs were found, with one correlating highly to the in vitro cultivated profile. The first novel program suggested a major metabolic shift, characterized by an active mitochondria and enhanced fatty acid metabolism. To understand the mechanism of the novel programs, parasite orthologues from published yeast data sets that had been generated under different experimental conditions were projected onto the parasite transcriptomes. This first novel state matched transcriptional patterns in yeast grown under starvation conditions and the second novel state resembled an environmental stress response. Interestingly, this latter parasite program had matched patient data demonstrating significantly higher temperatures, cytokines and markers of inflammation.

Could these novel states impact disease manifestations? The shift in metabolism could theoretically result in higher consumption of substrates and increase in metabolites such as lactic acid which could be injurious. The starvation like program also had a marked and coherent increase in abundance of genes encoding sexual forms which could result in enhanced transmission. The study did not have sufficient numbers of patients with clinically defined severe disease. However, patients expressing laboratory markers associated with severe disease were infected with parasites that had a similar second novel profile. These novel transcriptional patterns will need to be recapitulated in vitro to test their functional significance.

It is unknown if the second novel transcriptional program associated with patients demonstrating a significantly higher immune and inflammatory response encodes enhanced virulence. However, an increase in pathogen virulence as a consequence of host defense response has been described in Pseudomonas where exposure to interferon- $\gamma$ resulted in increased expression and function of pathogen virulence determinants (3). This scenario is reminiscent of game theory in which optimal behavior of one depends on the action of the other. We could speculate that the same scenario occurs in malaria where a robust immune response or inadequate substrates induce changes in parasite biology with heightened virulence or transmission.

The identification of novel in vivo parasite biology raises many new areas of exploration. Carrying out in vivo analysis is difficult, but can allow more accurate measurement of host pathogen biology that may be critical to understanding pathogenesis in this and other infections. - Johanna P. Daily.

\section{REFERENCES}

1. Llinas M, Bozdech Z, Wong ED, Adai AT, DeRisi JL 2006 Comparative whole genome transcriptome analysis of three Plasmodium falciparum strains. Nucleic Acids Res 34:1166-1173

2. Daily JP, Scanfeld D, Pochet N, Le Roch K, Plouffe D, Kamal M, Sarr O, Mboup S, Ndir O, Wypij D, Levasseur K, Thomas E, Tamayo P, Dong C, Zhou Y, Lander ES, Ndiaye D, Wirth D, Winzeler EA, Mesirov JP, Regev A 2007 Distinct physiological states of Plasmodium falciparum in malaria-infected patients. Nature 450:1091-1095

3. Wu L, Estrada O, Zaborina O, Bains M, Shen L, Kohler JE, Patel N, Musch MW, Chang EB, Fu YX, Jacobs MA, Nishimura MI, Hancock RE, Turner JR, Alverdy JC 2005 Recognition of host immune activation by Pseudomonas aeruginosa. Science 309:774-777 